\title{
Investigation on the Influence of Projectile with Different Diameter Base Cavity upon Its Flow Field
}

\author{
Yang Ni \\ Nanjing Artillery Academy, Nanjing, 211132, China \\ 598044525@qq.com
}

Keywords: Projectile with base cavity; Diameter; Flow field; Numerical simulation

\begin{abstract}
In order to discuss the influence of the base cavity diameter on the flow field of the projectile, projectile with different diameter base cavity $(118 \mathrm{~mm}, 116 \mathrm{~mm}, 114 \mathrm{~mm}, 112 \mathrm{~mm}, 110 \mathrm{~mm})$ is simulated. Flow field parameters and the aerodynamic drag coefficient of projectiles were obtained. The numerical results show that the projectile with smaller diameter base cavity has the smaller aerodynamic drag, but the difference is in apparent. The flow field of projectile with different diameter base cavity is similar, too.
\end{abstract}

\section{Introduction}

Upon the same condition, the range of fire for projectile with base cavity is farther than the projectile without base cavity 3 5\% [1]. Using base cavity configuration has been proved to be an effective method to increase the range of the gun by reducing the aerodynamic drag of the projectile[2-8].

In the present study, projectile with five kinds of different diameter base cavity is investigated numerically. The influence of the different diameter on the flow field and aerodynamic drag of the projectile is discussed.

\section{Numerical Method and Simulation Cases Setting}

Governing Equation The axisymmetric Navier-Stokes equation [9] and the $k$ - $\varepsilon$ turbulence model [10] are used in the simulation. The N-S equation is given by

$$
\partial U / \partial T^{+} \partial F(U) / \partial x+{ } \partial G(U) / \partial r+1 / r \cdot \partial H(U) / \partial r=1 / \operatorname{Re} \cdot\left(\partial F_{v}(U) / \partial x+{ }^{\partial} G_{v}(U) / \partial r+1 / r \cdot \partial H_{v}(U) / \partial r\right)
$$

where $U$ is the conservation variable, $F, G$ and $H$ are the inviscid terms, $F_{v}, G_{v}$ and $H_{v}$ are the viscous terms.

The convective terms are approximated using the Van Leer [11] splitting method and the central difference method is used for the viscous terms. The LU-SSOR scheme is used for the time integration.

Calculating Cases. As shown in Figure 1, the sketch map of the projectile with base cavity is given. In order to discuss the effect of the diameter of the base cavity on the flow field, projectiles with five different diameter (as shown in Table 1) base cavities are simulated in the paper.

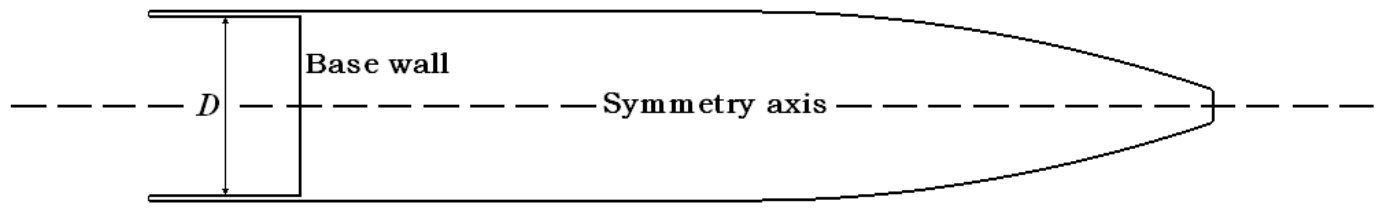

Figure 1. Schematic of the projectile

Table 1 Simulation cases

\begin{tabular}{|c|c|c|c|c|c|}
\hline Cases & 1 & 2 & 3 & 4 & 5 \\
\hline Diameter $[\mathrm{mm}]$ & 118 & 116 & 114 & 112 & 110 \\
\hline
\end{tabular}


Grid and Boundary Conditions. As shown in Fig. 2, the body-fitted grid of the projectile with base cavity (case 4 ) is given.
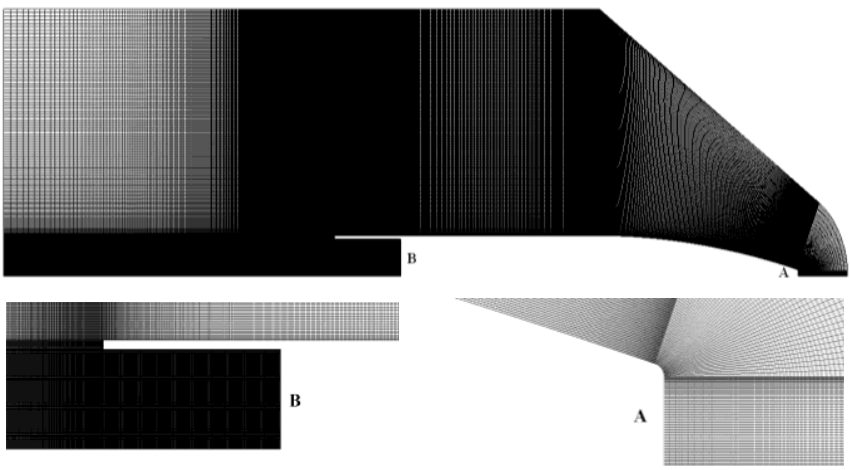

Figure 2. Grid of the simulation model (case 4)

The wall boundary condition is assumed to no-slip and adiabatic. The flow conditions are shown in Table 2.

Table 2 Boundary conditions

\begin{tabular}{|c|c|c|}
\hline Free stream parameter & Unit & Value \\
\hline Mach Number $(M a)$ & ---- & 1.97 \\
\hline Pressure $\left(p_{\infty}\right)$ & $\mathrm{Pa}$ & 101325 \\
\hline Temperature $\left(T_{\infty}\right)$ & $\mathrm{K}$ & 300 \\
\hline
\end{tabular}

\section{Results and Discussion}

Flow Field. The distributions of $M a$ contours and streamlines of the projectile with different diameter base cavity are shown in Fig. 3-Fig. 7. As show, the distributions of Ma contours and streamlines of projectiles are all similar. There is a classical detached bow shock wave formed in front of the projectile. At the bottom of the projectile, there is a fierce expansion wave and a large recirculation region is formed in the base cavity. With the reducing of the diameter of the base cavity, the thickness of the side wall of the cavity is increasing, the little circumfluence backside the side wall of the cavity become obvious. Fig. 8 shows the enlarged image of rear flow of projectile (Case 3). There is an obvious circumfluence backside the side wall.

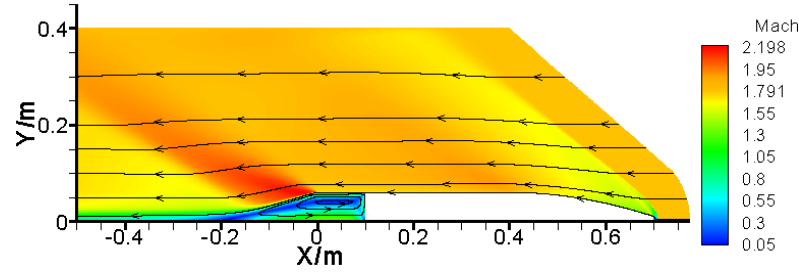

Figure 3. Ma (streamline) distribution of Case 1

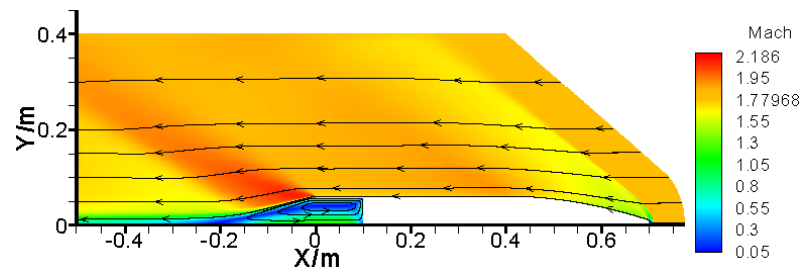

Figure 5. Ma (streamline) distribution of Case 3 Figure 6. Ma (streamline) distribution of Case 4

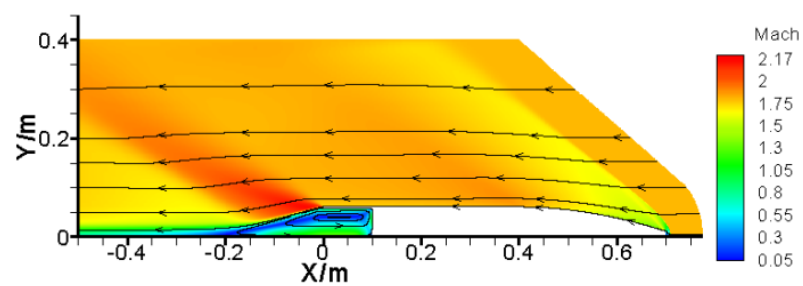

Figure 4. Ma (streamline) distribution of Case 2

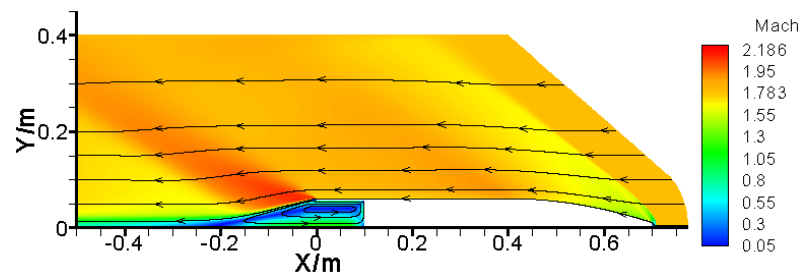



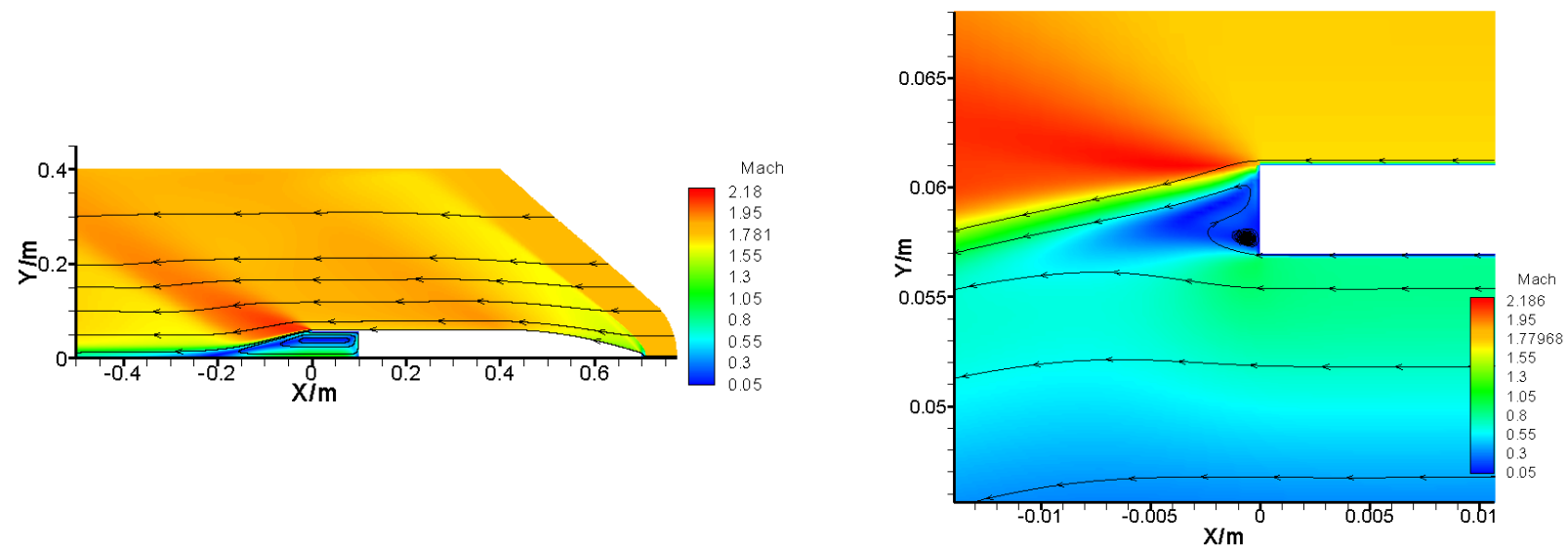

Figure 7. Ma (streamline) distribution of Case 5 Figure 8. Enlarged image of rear flow (Case 3)

The distribution of temperature and pressure of cases is similar too. As shown in Fig. 7, the distribution of temperature is given (Case 1 as example). The temperature maximum is located in the high temperature area which is formed inside the base cavity. The distribution of pressure is given (Case 5 as example) in Fig. 10. The pressure maximum is located at the stagnation point.

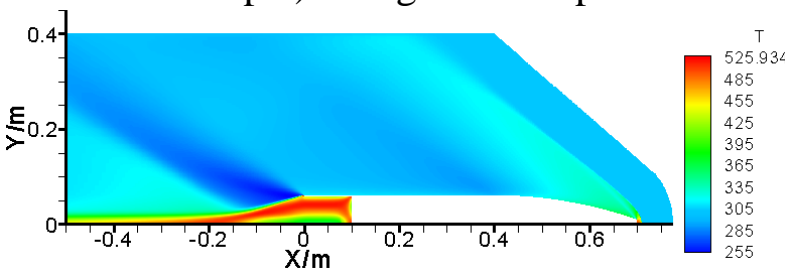

Figure 9. Temperature distribution (Case 1)

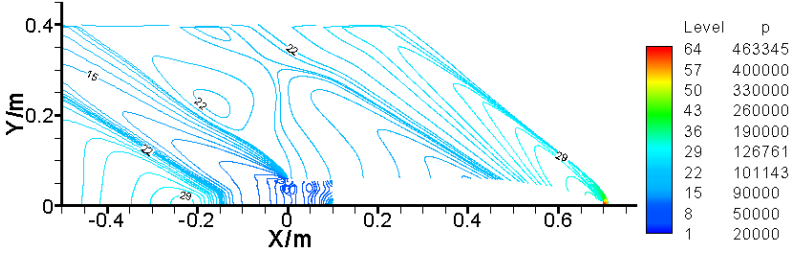

Figure 10. Pressure distribution (Case 5)

Aerodynamic Drag. The aerodynamic drag is one of the main parameter effective on the range of fire. The drag coefficient $\left(C_{d}\right)$ of the projectile is given by:

$$
C_{d}=F_{d} /\left(\frac{1}{2} \rho_{\infty} u_{\infty}^{2} \cdot S_{r e f}\right)
$$

where $F_{d}$ is the aerodynamic drug, $S_{r e f}$ is the reference area (the cross section of the projectile, diameter $122 \mathrm{~mm}$ ) .

As shown in Table 3, the aerodynamic drag coefficient of each case is given. With the reducing of the diameter of the base cavity, Case 1 to Case 5, the aerodynamic drag coefficient is reducing, too. And this amplitude of variation is no big.

Table 3 Aerodynamic drag coefficient of each case

\begin{tabular}{|c|c|c|c|c|c|}
\hline Case & 1 & 2 & 3 & 4 & 5 \\
\hline Drag coefficient $\left(C_{d}\right)$ & 0.3347 & 0.3267 & 0.3215 & 0.3178 & 0.3152 \\
\hline
\end{tabular}

\section{Conclusions}

Projectile with the different base cavity diameter $(118 \mathrm{~mm}, 116 \mathrm{~mm}, 114 \mathrm{~mm}, 112 \mathrm{~mm}$ and 110mm) was investigated numerically. The distribution of the flow field and the aerodynamic drag force of the projectile under a supersonic flow condition were obtained. The effect of the base cavity diameter on the projectile flow field and the aerodynamic force was discussed.

The flow field of the projectile has less affected by the value of the diameter of the base cavity.

The projectile with smaller diameter base cavity has the smaller aerodynamic drag. And this amplitude of variation is small, too. 
A smaller diameter base cavity is benefit to reduce the aerodynamic drag. But when the diameter of base cavity is smaller, the side wall of the base cavity becomes thicker. The mass of the projectile will be heavier obviously, this make against the increasing of the range of fire. So, the choice of the diameter of the base cavity for the projectile, which purpose to increase the range of fire, has to consider both the two factors, aerodynamic drag and the mass, comprehensively at least.

\section{References}

[1] H.Z. Wei, H.S. Zhu, The design theory of projectile, Beijing: National Defense Industry Press, 1985. (In Chinese)

[2] Z.Y. Wang, The effect about projectile of base cavity with side holes to flight drag under supersonic flow, ACTA Aerodynamic Sinica, 15(1997) 502-506.

[3] H.B. Lu, S.Y. Tian, The numerical investigation on flow field of projectile with base cavity, Advances in Engineering Research, 13 (2015) 250-251.

[4] J.Y. Cao, C.J. Lu, Y. Chen, X. Chen and J. Li, Research on the base cavity of a sub-launched projectile, Journal of Hydrodynamics, 24(2012) 244-249.

[5] J. Howell, D. Sims-Williams, A. Sprot, F. Hamlin and R. Dominy, Bluff body drag reduction with ventilated base cavities, SAE Technical Papers, 5(2012) 152-160.

[6] M. G. Edgar, Preliminary investigation of effectiveness of base bleeds in reducing drag of blunt-base bodies in supersonic stream, NASA RM26, 1971.

[7] Ibrahim, A. Filippone, Supersonic aerodynamics of a projectile with slot cavities, Aeronautical Journal, 114(2010) 15-24.

[8] R. D. Elizabeth, The effectiveness of base-bleed in reducing drag of boat-tailed bodies at supersonic velocities, PB157711, 1970.

[9] W.Q. Tao, Numerical Heat Transfer, 2nd ed. Xi' an: Xi'an Jiaotong University Press, 2001 (In Chinese)

[10] X.S. Wu, The numerical investigation on flow field of projectile (with base bleed), Journal of ballistics, 4 (1992) 39-43. (In Chinese)

[11] X.D. Wang, J.J. Tan, X.H. Lin and Z.H. Tang, Research on parallel numerical simulation of N-S equations based on Van Leer+AUSM scheme, Journal of Astronautics, 31(2010) 986-992. (In Chinese) 